\title{
Analysis of 6-methoxy podophyllotoxin and podophyllotoxin in hairy root cultures of Linum album Kotschy ex Boiss
}

\author{
Najmeh Ahmadian Chashmi • Mozafar Sharifi • \\ Morteza Yousefzadi • Mehrdad Behmanesh • \\ Hassan Rezadoost • Alejandra Cardillo · Javier Palazon
}

Received: 23 January 2012/ Accepted: 18 April 2012

(C) Springer Science+Business Media, LLC 2012

\begin{abstract}
Linum album is a herbaceous medicinal plant that contains some lignans with antiviral and anticancer properties such as podophyllotoxin (PTOX) and 6-methoxy podophyllotoxin (MPTOX). In this research, hairy root cultures of $L$. album were established by transformation with Agrobacterium rhizogenes strains LBA9402, A4, AR15834 and Agrobacterium tumefaciens strain C58C1 (pRiA4). The presence of PTOX and MPTOX in the hairy
\end{abstract}

Electronic supplementary material The online version of this article (doi:10.1007/s00044-012-0067-1) contains supplementary material, which is available to authorized users.

N. A. Chashmi · M. Sharifi $(\bowtie)$

Department of Plant Biology, Faculty of Biological Sciences,

Tarbiat Modares University, P.O. Box: 14115-154, Tehran, Iran

e-mail: msharifi@modares.ac.ir

M. Yousefzadi

Department of Biology, Faculty of Sciences, Hormozgan

University, Bandar Abbas, Iran

M. Behmanesh

Department of Genetics, Faculty of Biological Sciences,

Tarbiat Modares University, Tehran, Iran

H. Rezadoost

Department of Phytochemistry, Medicinal Plants and Drugs

Research Institute, Shahid Beheshti University, Tehran, Iran

A. Cardillo

Catedra de Microbiología Industrial y Biotecnología,

Facultad de Farmacia y Bioquímica, Universidad de Buenos

Aires, Buenos Aires, Argentina

J. Palazon

Laboratori de Fisiologia Vegetal, Facultat de Farmacia,

Universitat de Barcelona, Barcelona, Spain roots was verified by ESI/MS in positive ion mode. MPTOX was confirmed and its enantiomer determined by nuclear magnetic resonance spectroscopy and circular dichroism spectroscopy, respectively. PTOX and MPTOX production was determined by HPLC, in different lines of hairy roots. The results showed that all obtained hairy root lines produced higher yield of lignans than mother plant roots. In addition, the lignan content in the roots derived from A. rhizogenes strain LBA9402 was higher than in those obtained from A. tumefaciens strain C58C1.

Keywords Agrobacterium - Hairy roots - Linum album . Podophyllotoxin (PTOX) .

6-methoxy podophyllotoxin (MPTOX)

$\begin{array}{ll}\text { Abbreviations } & \\ \text { Ags } & \text { Agropine synthase } \\ \text { CD } & \begin{array}{l}\text { Circular dichroism } \\ \text { Liquid chromatography-electrospray- } \\ \text { mass spectrometry/mass spectrometry }\end{array} \\ \text { MPTOX } & \begin{array}{l}\text { 6-methoxy podophyllotoxin } \\ \text { Nuclear magnetic resonance }\end{array} \\ \text { NMR } & \text { Podophyllotoxin }\end{array}$

\section{Introduction}

Lignans constitute a large group of secondary metabolites synthesized by many plants. These compounds are usually formed from two phenylpropanoid units and manifest considerable biological activity (Smollny et al., 1998). Podophyllotoxin (PTOX), a lignan with antiviral and antineoplastic activities, is used today primarily as a 
precursor for the semi-synthesis of established cancer therapeutics such as etoposide, teniposide and etopophos (Gordaliza et al., 2004). To date, extracting PTOX and 6-methoxy podophyllotoxin (MPTOX) from the subterrestrial parts of Podophyllum species is still preferred to their chemical synthesis due to low product yields and the complexity of the enantioselective reactions (Botta et al., 2001). Plants from the genus Linum (the family Linaceae), with their ability to synthesize and accumulate lignans in callus and hairy root cultures, provide an additional source of PTOX and MPTOX (Farkya and Bisaria, 2008).

Biotechnological methods are being increasingly studied for their application as alternative ways of obtaining these valuable metabolites (Ramachandra and Ravishankar, 2002), although only a few have so far been produced at an industrial level owing to certain limitations (Chattopadhyay et al., 2002; Zhong, 2002). As suspension cultures are not genetically stable (Bais et al., 2001), new methods have been sought to improve lignan production. Hairy roots are transformed roots derived from the infection of wounded plant tissue by the soil bacterium, Agrobacterium rhizogenes. Such hairy roots often grow as fast as plant cell cultures or faster, and do not require hormones in the growth medium (Giri and Narasu, 2000). The greatest advantage of using hairy roots is that their biosynthetic capacity for metabolite production is similar to that of the mother plants or greater. Most of the research on the production of PTOX and related lignans has used undifferentiated cell cultures derived from non-transformed explants of naturally growing plants or aseptically growing seedlings (Smollny et al., 1998; Farkya et al., 2004; Kuhlmann et al., 2002; Nikolay and Ionkova, 2005). The in vitro production of PTOX in Podophyllum hexandrum has been reported at the bioreactor level under batch, fed-batch and continuous modes with optimized medium conditions only in non-transformed undifferentiated cell suspension cultures (Chattopadhyay et al., 2002, 2003a, b, c). Transformed calli of $P$. hexandrum obtained by infecting its embryos with $A$. rhizogenes, in the presence of phytohormones, showed a threefold increase in PTOX content compared with non-transformed cell cultures (Giri et al., 2001).

In this study, hairy root cultures were established by infecting derived leaf segments of L. album with A. rhizogenes strains LBA9402, A4, AR15834 and A. tumefaciens strain C58C1 (pRiA4). PTOX and MPTOX production in two lines of hairy roots was compared. The presence of PTOX and MPTOX in the samples was verified by ESI/MS in positive ion mode. In order to confirm MPTOX and determine its enantiomer, nuclear magnetic resonance (NMR) spectroscopy and circular dichroism (CD) spectroscopy were used, respectively.

\section{Experimental}

Plant material

Linum album seeds were collected from Sohanak, $\left(35^{\circ} 48^{\prime}\right.$ $\mathrm{N}, 51^{\circ} 32^{\prime} \mathrm{E}$ and altitude of $1,900 \mathrm{~m}$ ) Iran. The plant material was kindly identified by Dr. Shahrokh Kazempour (Tarbiat Modares University, Tehran, Iran) and a voucher specimen was deposited at the herbarium of the Biological Sciences Faculty, Tarbiat Modares University, Tehran, Iran. The seeds were surface-sterilized with $70 \%(\mathrm{v} / \mathrm{v})$ ethanol for $70 \mathrm{~s}$, and $2 \%(\mathrm{v} / \mathrm{v})$ sodium hypochlorite for $20 \mathrm{~min}$, and then were washed with sterile distilled water more than three times. Sterile seeds were treated with $\mathrm{GA}_{3}$ (500 $\mathrm{mg} \mathrm{L}^{-1}$ ) overnight, then cultured on hormone-free MS medium supplemented with sucrose (3\%) (Murashige and Skoog, 1962) and incubated at $25^{\circ} \mathrm{C}$ with a $16-\mathrm{h}$ photoperiod. Sterile grown seedlings were used to initiate cultures.

\section{Agrobacterium culture}

Agrobacterium rhizogenes strains LBA9402, A4, AR15834 and A. tumefaciens C58C1 (pRiA4) were used for infections. The bacteria from glycerol stock (stored at $-80{ }^{\circ} \mathrm{C}$ ) were cultured on LB solid medium (Park and Facchini, 2000) containing $50 \mathrm{mg} \mathrm{L}^{-1}(\mathrm{w} / \mathrm{v})$ rifampicin for screening and selecting the desired colony. Bacterial suspensions were incubated in liquid LB medium for 2 days at $28^{\circ} \mathrm{C}$.

\section{Plant transformation}

Two procedures were tested for initiation of L. album hairy roots: the syringe needle and the leaf disk methods (Lin et al., 2003). In the first procedure, separate syringe needles were contaminated with Agrobacteria colonies strains LBA9402, A4, AR15834 and C58C1 (pRiA4) and used to infect 4-week-old L. album seedlings by pricking the leaves and stems, and then placed on solid MS medium as described above. Also, seedlings pricked with a sterile syringe needle, without infection, were used as the control. After incubation in the dark at $25^{\circ} \mathrm{C}$ for 2 days, the infected seedlings were placed on solid MS medium containing $200 \mathrm{mg} \mathrm{L}^{-1}$ cefotaxime antibiotic and incubated at $25^{\circ} \mathrm{C}$.

In the leaf disk method, leaves of $L$. album seedlings were excised and cut in half transversally. The leaf disks were immersed in the $A$. rhizogenes suspension for $10 \mathrm{~min}$, blotted to remove excess bacteria, and placed on solid MS medium as described above. Uninfected sterile leaf explants were grown on the MS medium culture as the control. The obtained hairy roots were cultured in MS medium by serial sub-culturing every 2 weeks. 
PCR analysis

DNA was extracted from non-transformed root, obtained from seedlings, and transformed hairy roots using the acetyl trimethyl ammonium bromide (CTAB) method (Khan et al., 2007). The transgenic nature of hairy roots was confirmed by PCR rolC, Ags (agropine synthase) and VirD specific primers. Sequences of these primers are shown in Table 1. Amplification conditions for rolC, Ags and VirD were 1 cycle at $94{ }^{\circ} \mathrm{C}$ for $2 \mathrm{~min}$ followed by 35 cycles of amplification $\left(45 \mathrm{~s}\right.$ at $94{ }^{\circ} \mathrm{C}, 40 \mathrm{~s}$ at $55^{\circ} \mathrm{C}$ and $45 \mathrm{~s}$ at $72{ }^{\circ} \mathrm{C}$ ) and 1 cycle at $72{ }^{\circ} \mathrm{C}$ for $10 \mathrm{~min}$. PCR products were analyzed by electrophoresis in a $1 \%(\mathrm{w} / \mathrm{v})$ agarose-ethidium bromide gel. Meanwhile, the Ri-plasmid of $A$. rhizogenes was used as a positive control (Wang et al., 2006).

Growth kinetics and biomass measurements

The growth rate was measured as hairy root growth kinetics at the first of the experiment. The experimental conditions were as follows. Hairy root lines with $2.4 \mathrm{~g}$ of root mass (on fresh weight basis) were transferred to a 250-ml Erlenmeyer flask containing $50 \mathrm{ml}$ of hormone-free MS liquid medium at $25{ }^{\circ} \mathrm{C}$ for period of 4 weeks. Flasks were harvested with three replications each week (up to day 28 after inoculation). The growth of hairy root lines was measured every week. Hairy roots were dried by blotting paper to remove all external moisture and weighed to determine the final fresh weight (FW) before being frozen for lignan extraction.

\section{Extraction of lignans}

Powdered hairy roots $(2 \mathrm{~g})$ were extracted by sonication in methanol $(80 \%)$ during $1 \mathrm{~h}$. Dichloromethane and water $(1: 1 \mathrm{v} / \mathrm{v})$ were added to obtain a partition of compounds between two layers. The dichloromethane fractions were collected, dried and dissolved in $500 \mu$ of HPLC grade methanol and then injected into the HPLC (Van Uden et al., 1989) (Philips, UV/Vis detector; $\mathrm{Pu}$ 41110). The presence of PTOX and MPTOX in the hairy roots was determined by ESI/MS in positive ion mode and $0.05 \%$ formic acid was used.

\section{MPTOX purification and confirmation}

Analytical separation was carried out to confirm MPTOX and determine its enantiomer. Separations were performed on a C18-S5ODS3 $(250 \times 4.6 \mathrm{~mm}) \mathrm{RP}$ column with a flow rate of $1.0 \mathrm{ml} \mathrm{min}{ }^{-1}$. A fraction containing MPTOX was collected, lyophilized and dissolved in DMSO and subjected to $500 \mathrm{MHz}$ NMR spectroscopy. A NMR spectrum was recorded on Bruker DRX500 Avance running XWINNMR 2.6 software using $500 \mu \mathrm{l}$ samples. The chemical shifts (delta) are reported in parts per million (ppm). Also, FT-RI was carried out with a Bruker tensor 27 spectrometer.

The CD spectrum was obtained with a JASCO J-715 spectropolarimeter using cuvettes with optical lengths of $1 \mathrm{~cm}$ and detection at 250-350 $\mathrm{nm}$. The final data were obtained by subtracting the buffer contribution (DMSO) from the original spectrum.

Optical rotation $\left([\alpha]_{\mathrm{D}}\right.$ values) of MPTOX was measured on a Perkin Elmer 241 automatic polarimeter (Überlingen, Germany) at $20{ }^{\circ} \mathrm{C}$. The specific rotation $\left([\alpha]_{\mathrm{D}}\right)$ at the sodium D line $(\lambda 589 \AA)$ was calculated as follows:

$[\alpha]_{\mathrm{D}}=(100 \times \alpha) /(l \times c), \alpha=$ observed angle of rotation, $l=$ length of the path of light through the solution in decimeters $(\mathrm{dm}), c=$ concentration of the solution in $\mathrm{g} / 100 \mathrm{ml}$.

\section{Analysis of lignans}

PTOX and MPTOX quantification was analyzed using HPLC as described previously by Yousefzadi et al. (2010b). The stationary phase was a C18-ODS3, $5 \mu \mathrm{m}$ (250-4.6 mm) column. The elution solvent was composed of water and acetonitrile with a gradient system. This system with acetonitrile $(\mathrm{A} \%)$ and water $(\mathrm{B} \%)$ was used as follows: (0 min, flow rate 0.8): A (40), B (33); (10 min, flow rate 1): A (67), B (33); (17 min, flow rate 1): A (40), B
Table 1 Sequences of used primers

\begin{tabular}{llc}
\hline Genes & Sequences & $\begin{array}{l}\text { Amplicon } \\
\text { size (bp) }\end{array}$ \\
\hline rolC & Forward primer 5'-TAACATGGCTGAAGACGACC-3' & 534 \\
& Reverse primer 5'-AAACTTGCACTCGCCATGCC-3' \\
Ags & Forward primer 5'-GGCGTGAGCACCTCATATCCG-3' \\
& Reverse primer 5'-TTCGAAGCCTTTGCCTGCAAA-3' & \\
\multirow{2}{*}{ virD } & Forward primer 5'-ATGTCGCAAGGCAGTAAGCCC-3' & 347 \\
& Reverse primer 5'-GGAGTCTTTCAGCATGGAGCAA-3' & \\
& & 438 \\
\hline
\end{tabular}


(60); (21 min, flow rate 0.8): A (40), B (60). A spectrophotometric UV detection was used $(290 \mathrm{~nm})$. The calibration was performed with standard PTOX (RT= $9.5 \pm 0.1 \mathrm{~min})$ and $\operatorname{MPTOX}(R T=11.5 \pm 0.1 \mathrm{~min})$ (Fig. 1). Every sample was assayed with three replications. The lignan production was determined using a calibration curve compared with standards and a co-chromatogram of the standards and samples.

\section{Statistical analysis}

All data were analyzed using MSTAT-C software. Duncan's multiple range tests were used to show statistical differences between treatment methods and controls. $P \leq 0.05$ or $\leq 0.01$ was considered significantly different.
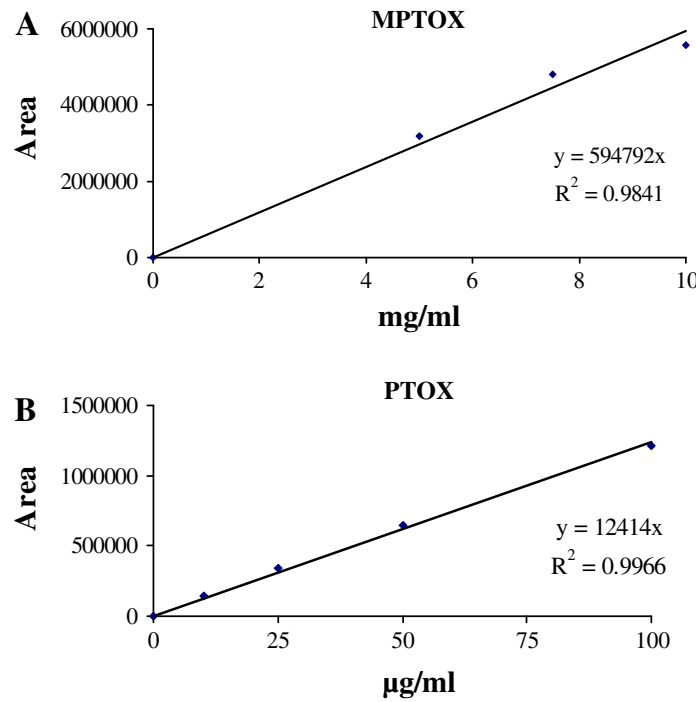

Fig. 1 6-Methoxy podophyllotoxin (a) and podophyllotoxin (b) calibration curve using HPLC. Each value is the mean of three replications

\section{Results and Discussion}

L. album hairy root induction

Although all four Agrobacterium strains applied in this work are among the most virulent in common usage (Lin et al., 2003), despite several attempts using different bacterial strains and infection sites, no hairy roots were obtained using the leaf disk infection technique. Infection by syringe needle was more successful, with strains LBA9402 and C58C1 (pRiA4) showing their rooting ability, the former more readily. LBA9402 hairy roots of $L$. album were induced after 4 weeks of infection and had a higher transformed percentage $(20 \%)$, while C58C1 (pRiA4) hairy roots were induced after 5 weeks with a lower percentage of transformation $(10 \%$ ) (data are not shown). Some transgenic roots turned brown and aged considerably faster than others. These brown roots were discarded and only those showing a good growth capacity were maintained for further characterization. Although various explant sources can be used to produce hairy roots in vitro, in some cases specific tissues perform better than others (Mishra et al., 2011). Regarding L. album, young stems produced more primary cultures than other tissues.

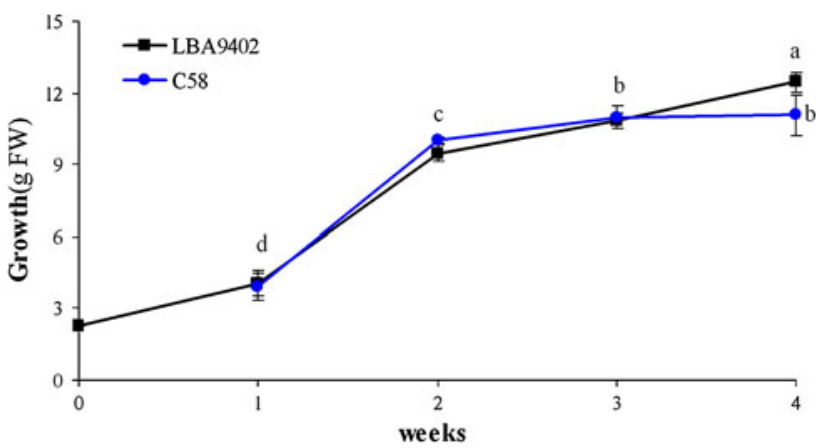

Fig. 2 Growth pattern of hairy roots induced by A. rhizogenes strains LBA9402 and A. tumefactions C58C1 (pRiA4). Data represent average values from four separate experiments \pm SD
Fig. 3 Electrophoretogram showing the presence of $\mathrm{rolC}$ (a), Ags (b) genes and absence of $\operatorname{VirD}(\mathbf{c})$ gene $(534,347$, 483 bp bands, respectively) in transformed hairy roots; $M$ molecular weight marker, $P C$ positive control, R1 and R2: transformed root lines LBA9402 and C58C1, respectively, $\mathrm{R}^{-}$: the nontransformed root

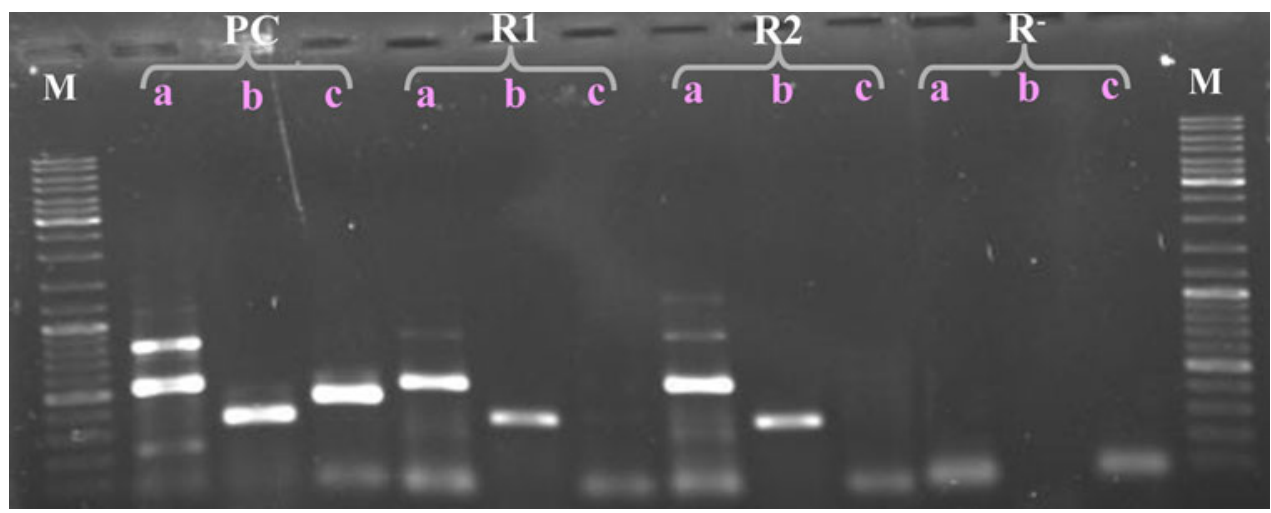


Efficiency of transformation differed among the Agrobacterium strains, as reported by other authors (Giri et al., 2001; Mishra et al., 2011).

A time course experiment (up to 4 weeks at defined time intervals) was performed to monitor root growth and biomass production and also to estimate lignan content. The growth kinetics of L. album hairy roots showed three different phases: the lag phase (first week) when the specific growth rate is essentially slow, the exponential growth phase (second and third weeks) when the specific growth rate is constant and the stationary phase (fourth week) with the emergence of at least one limiting parameter. The highest hairy root growth was recorded with strain LBA9402 (12.455 g FW) after 4 weeks of culture (Fig. 2). Hairy roots induced by $\mathrm{C} 58 \mathrm{C} 1$ (pRiA4) showed comparatively slow growth (11.083 g FW). However, transformed hairy roots induced by both strains exhibited negative geotropism. The transformed roots with A. rhizogenes have
Fig. 4 CD spectrum of MPTOX. Detected solvents: grade, detected temperature: $25{ }^{\circ} \mathrm{C}$. UV detected wavelength at $250-350 \mathrm{~nm}$ methanol of chromatographic

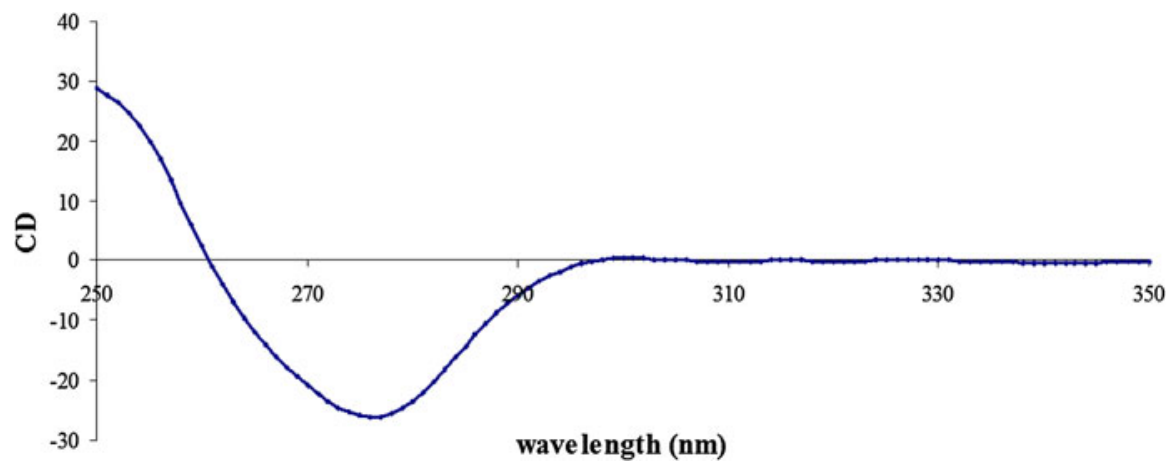

Fig. 5 Selected molecular ion $(445 \mathrm{~m} / \mathrm{z})$ in TIC (up) and mass spectrum of 6-MPTOX (down)
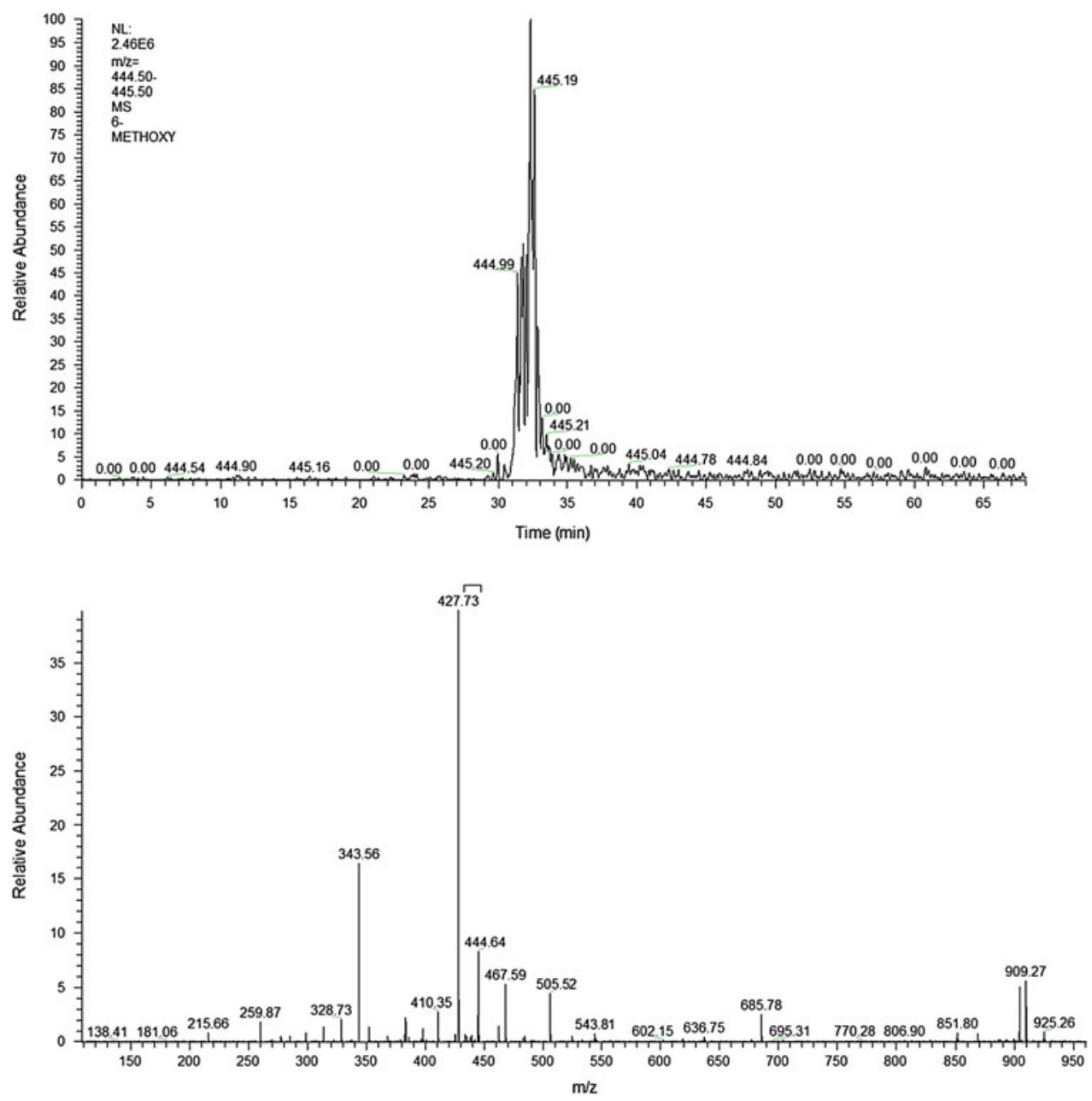
an altered phenotype, showing profuse lateral growth, lack of geotropism and fast growth in the culture (Mishra et al., 2011).

\section{Confirmation of transformation}

In order to assess the genetic status of the hairy roots, we used a PCR-based analysis that targeted rolC, Ags (opine synthase) and virD genes. The rolC and $A g s$ genes are diagnostic for T-DNA integration into the host genome. The virD gene, located outside the T-DNA, is diagnostic for the presence of any remaining Agrobacteria in the root tissue. PCR analysis revealed the presence of the rolC and Ags genes in hairy roots, indicating that the T-DNA was retained in hairy roots (Fig. 3). Confirmation of contamination was also done by PCR analysis using virD (483 bp) gene-specific primers. It was not found in the hairy root lines, thus indicating the absence of contamination with Agrobacterium strains LBA9402 and C58C1. Also, rolC, Ags and virD genes were not detected in the non-transformed root.

\section{Confirmation of MPTOX and PTOX}

\section{Identification of MPTOX in transformed root cultures}

Lignan (dimmers of monolignols) formation and lignin (polymers of monolignols) biosynthesis are catalyzed by different enzymes, and a consequence is that natural lignans are normally enantiomerically pure because they arise from stereochemically controlled coupling (Dewik, 2002). For example, the lignan pinoresinol, and its derived matairesinol, was found only as their (-)-isomers in suspension-cultured Pinus taeda (Eberhardt et al. 1993). Data acquired using a polarimeter (optical rotation $=[\alpha]_{\mathrm{D}}-$ $\left.60^{\circ}\right)$ and $\mathrm{CD}$ spectrum confirmed the presence of the (-)isomer of MPTOX (Fig. 4).

Moreover, this less hydrophilic compound was identified by NMR and IR spectroscopy. Significant ${ }^{1} \mathrm{H}-\mathrm{NMR}$ data are shown in the following order: chemical shift $(\delta)$ expressed in ppm, multiplicity (s, singlet; d, doublet; $\mathrm{m}$, multiplet and $\mathrm{ABq}, \mathrm{AB}$ quartet).

${ }^{1} \mathrm{H}-\mathrm{NMR}(500 \mathrm{MHz},(\mathrm{CD} 3) 2 \mathrm{SO}): \delta=6.39(2 \mathrm{H}, \mathrm{s})$, $6.29(1 \mathrm{H}, \mathrm{s}), 5.97(2 \mathrm{H}, \mathrm{d}), 5.04(1 \mathrm{H}, \mathrm{d}), 4.93(1 \mathrm{H}, \mathrm{m}), 4.45$ (2 H, ABq), $4.08(1 \mathrm{H}, \mathrm{dd}), 3.95\left(3 \mathrm{H}, \mathrm{s}, \mathrm{OCH}_{3}\right), 3.62(6 \mathrm{H}, \mathrm{s}$, $\left.\left(\mathrm{OCH}_{3}\right) 2\right), 3.6\left(3 \mathrm{H}, \mathrm{s}, \mathrm{OCH}_{3}\right), 3.0(1 \mathrm{H}, \mathrm{dd})$. Melting point: 80-83. IR: 3449.20, 2924.41, 2862.45, 1775.34, 1634.25, 1465.59, 1247.14, 1124.43, 1050.77 .

\section{MS analyses of MPTOX}

For identification, liquid chromatography-electrospraymass spectrometry/mass spectrometry (LC-ESI-MS/MS) analysis was carried out. Several reports have been cited in the literature demonstrating the applicability of MS and MS/MS coupled with liquid chromatography in the
Fig. 6 The fragmentation of MPTOX (according to Li et al. 2007)

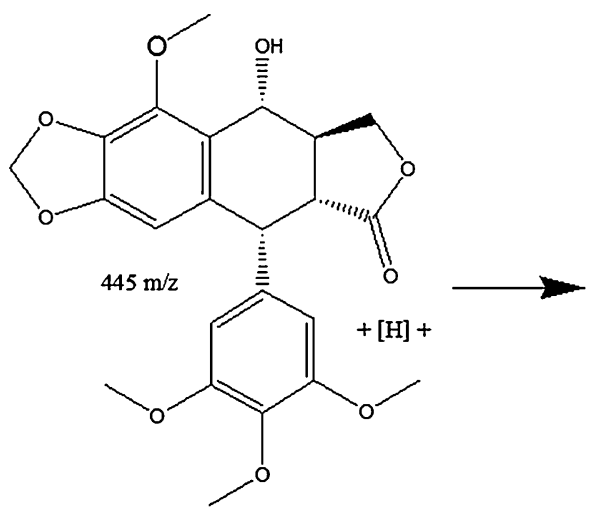<smiles>COc1cc([C@@H]2c3cc4c(c(OC)c3C=C3COC(=O)[C@H]32)OCO4)cc(OC)c1OC</smiles> 
analyses of PTOX and its derivatives (Li et al., 2007; Lin et al., 2008). In this work, $[\mathrm{M}+\mathrm{H}]^{+}$and $[\mathrm{M}+\mathrm{Na}]^{+}$ions of 6-MPTOX were determined. According to Fig. 5, the observed mass at $467 \mathrm{Da}$ corresponds to the $[\mathrm{M}+\mathrm{Na}]^{+}$ ion of 6-MPTOX and the observed mass of $445 \mathrm{Da}$ is related to the $[\mathrm{M}+\mathrm{H}]^{+}$ion of 6-MPTOX. The other peaks at mass of 427 and 343 Da correspond to fragments illustrated in Fig. 6.

\section{Confirmation of PTOX}

The presence of PTOX in the hairy roots was verified by ESI/MS in positive ion mode, as previously described (Yousefzadi et al., 2010a).

\section{Quantification of lignans}

Among the examined samples, hairy roots induced by strain LBA9402 were found to be the best in terms of biomass as well as lignan production. The maximum concentrations of PTOX (105 $\left.\mu \mathrm{g} \mathrm{g}^{-1} \mathrm{DW}\right)$, and 6-MPTOX (48 $\mathrm{mg} \mathrm{g}^{-1} \mathrm{DW}$ ) were recorded in hairy roots induced by strain LBA9402 (Fig. 7). In the roots of mother plants which were collected from its natural habitat, concentrations of PTOX and 6-MPTOX were 14.11 and $1.13 \mathrm{mg}$ $\mathrm{g}^{-1} \mathrm{DW}$, respectively. When compared to the roots of
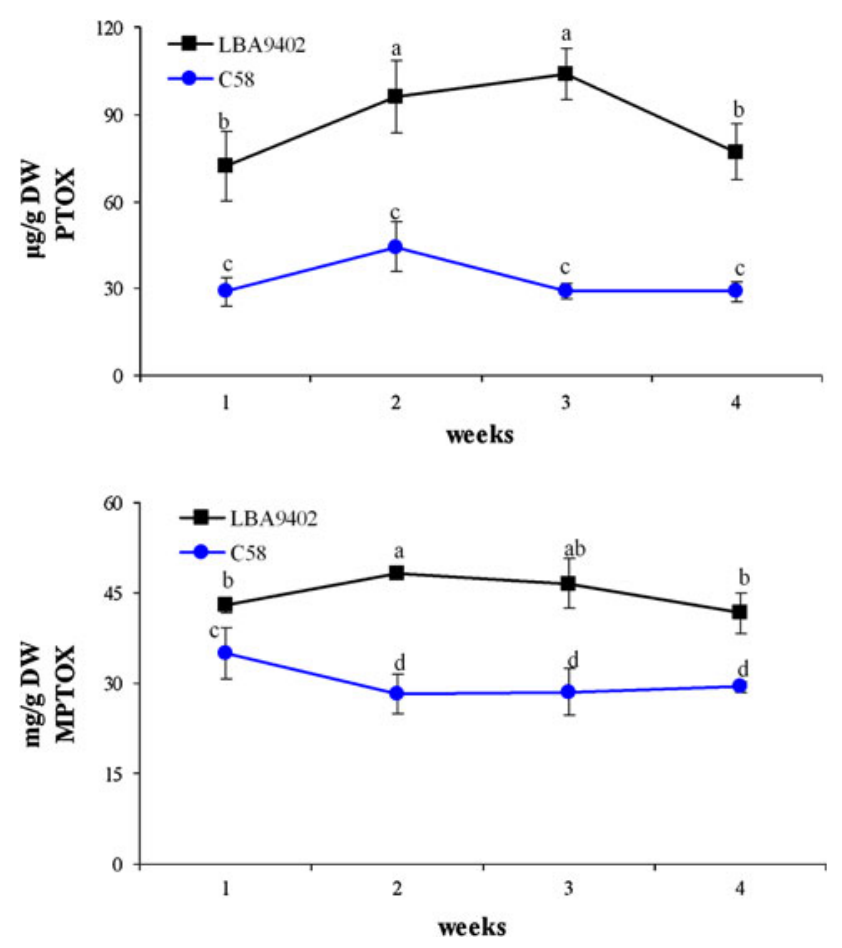

Fig. 7 PTOX and MPTOX production in a 4-week period. PTOX: podophyllotoxin. MPTOX: 6-methoxy podophyllotoxin. Data represent average values from three separate experiments \pm SD mother plant, the hairy root cultures were found to contain higher concentrations of lignans. This confirms that the insertion sites of Ri-T-DNA interfere with the secondary metabolite biosynthetic pathway of lignans. Similar results have been reported by several groups (Mishra et al., 2011; Verma et al., 2007).

\section{Conclusion}

In general, the amount of anti-cancer lignans (PTOX and MPTOX) in plants is considerably less than in transformed hairy roots. In addition, the hairy roots exhibited more rapid growth than plants. This study may open possibilities for bioreactor-based production of the pharmaceutically important metabolites PTOX and 6-MPTOX.

\section{References}

Bais HP, Sudha G, George J, Ravishankar GA (2001) Influence of exogenous hormones on growth and secondary metabolite production in hairy root cultures of Cichorium intybus L. Cv. Lucknow local. In Vitro Cell Dev Biol Plant 37:293-299

Botta B, Delle Monache G, Misiti D, Vitali A, Zappia G (2001) Aryltetralin lignans: chemistry, pharmacology and biotransformation. Curr Med Chem 8:1363-1381

Chattopadhyay S, Srivastava AK, Bisaria VS (2002) Optimization of culture parameters for production of podophyllotoxin in suspension culture of Podophyllum hexandrum. Appl Biochem Biotechnol 102:381-393

Chattopadhyay S, Bisaria VS, Srivastava AK (2003a) Enhanced production of podophyllotoxin by Podophyllum hexandrum using in situ cell retention bioreactor. Biotechnol Prog 19:1026-1028

Chattopadhyay S, Bisaria VS, Bhojwani SS, Srivastava AK (2003b) Enhanced production of podophyllotoxin by fed-batch cultivation of Podophyllum hexandrum. Can J Chem Eng 81:1-8

Chattopadhyay S, Farkya S, Srivastava AK, Bisaria VS (2003c) Bioprocess considerations for production of secondary metabolites by plant cell suspension cultures. Biotechnol Bioprocess Eng 7:138-149

Dewik PM (2002) Medicinal natural products, a biosynthetic approach. Wiley, Chichester, pp 121-136

Eberhardt TL, Bernards MA, He L, Davin LB, Wooten JB, Lewis NG (1993) Lignification in cell suspension cultures of Pinus taeda: in situ characterization of a Gymnosperm lignin. J Biol Chem 268:21088-21096

Farkya S, Bisaria VS (2008) Exogenous hormones affecting morphology and biosynthetic potential of hairy root line (LYR2i) of Linum album. J Biosci Bioeng 105:140-146

Farkya S, Bisaria VS, Srivastava AK (2004) Biotechnological aspects of the production of the anticancer drug podophyllotoxin. Appl Microbiol Biotechnol 65:504-519

Giri A, Narasu ML (2000) Transgenic hairy roots: recent trends and applications. Biotechnol Adv 18:1-22

Giri A, Giri CC, Dhingra V, Narasu ML (2001) Enhanced podophyllotoxin production from Agrobacterium rhizogenes transformed cultures of Podophyllum hexandrum. Nat Prod Lett $15: 229-235$ 
Gordaliza M, Garc PA, del Corral M, Castro MA, Gomez-Zurita MA (2004) Podophyllotoxin: distribution, sources, applications and new cytotoxic derivatives. Toxicon 44:441-459

Khan S, Irfan Qureshi M, Kamaluddin Alam T, Abdin MZ (2007) Protocol for isolation of genomic DNA from dry and fresh roots of medicinal plants suitable for RAPD and restriction digestion. Afr J Biotechnol 6:175-178

Kuhlmann S, Kranz K, Lücking B, Alfermann AW, Petersen M (2002) Aspects of cytotoxic lignan biosynthesis in suspension cultures of Linum nodiflorum. Phytochem Rev 1:37-43

Li Q, Yan G, Ge T (2007) A fragmentation study of podophyllotoxin and its 40-demethyl-4b-substituted derivatives by electrospray ionization ion-trap time-of-flight tandem mass spectrometry. Rapid Commun Mass Spectrom 21:2843-2852

Lin H, Kwok KH, Doran PM (2003) Development of Linum flavum hairy root cultures for production of Coniferin. Biotechnol Lett 25:521-525

Lin MC, Lin JH, Chen SK, Cheng YW, Cheng HW (2008) Simultaneous determination of podophyllotoxin, quercetin and kaempferol in podophyllin by liquid chromatography tandem mass Spectrometry. J Food Drug Anal 16:29-40

Mishra J, Bhandari H, Singh M, Rawat S, Agnihotri RK, Mishra S, Purohit S (2011) Hairy root culture of Picrorhiza kurroa Royle ex Benth.: a promising approach for the production of picrotin and picrotoxinin. Acta Physiol Plant 33(5):1841-1846

Murashige T, Skoog F (1962) A revised medium for rapid growth and bioassays with tobacco tissue cultures. Plant Physiol 15:473-497

Nikolay V, Ionkova I (2005) Lignan production by cell cultures of Linum setaceum and Linum campanulatum. Pharm Biol 43:509-511

Park S, Facchini P (2000) Agrobacterium rhizogenes-mediated transformation of opium poppy, Papaver somniferum L., and
California poppy, Escholizia californica cham., root cultures. J Exp Bot 51:1005-1016

Ramachandra RS, Ravishankar GA (2002) Plant cell cultures: chemical factories of secondary metabolites. Biotechnol Adv 20:101-153

Smollny T, Wichers H, Kalenberg S, Shahsavari A, Petersen M, Alfermann WA (1998) Accumulation of podophyllotoxin and related lignans in cell suspension cultures of Linum album. Phytochemistry 48:975-979

Van Uden W, Pras N, Visser JF, Malingre TM (1989) Detection and identification of podophyllotoxin produced by cell cultures derived from Podophyllum hexandrum Royle. Plant Cell Rep 8:165-168

Verma PC, Rahman L, Negi AS, Jain DC, Khanuja SPS, Banerjee S (2007) Agrobacterium rhizogenes mediated transformation of Picrorhiza kurroa Royle ex Benth.: establishment and selection of superior hairy root clone. Plant Biotechnol Rep 1:169-174

Wang B, Zhang G, Zhu L, Chen L, Zhang Y (2006) Genetic transformation of Echinacea purpurea with Agrobacterium rhizogenes and bioactive ingredient analysis in transformed cultures. Colloid Surface B 53:101-104

Yousefzadi M, Sharifi M, Behmanesh M, Ghasempour A, Moyano E, Palazon J (2010a) Salicylic acid improves podophyllotoxin production in cell cultures of Linum album by increasing the expression of genes related with its biosynthesis. Biotechnol Lett 32:1739-1743

Yousefzadi M, Sharifi M, Ahmadian Chashmi N, Behmanesh M, Ghasempour A (2010b) Optimization of podophyllotoxin extraction method from Linum album cell cultures. Pharm Biol 48:1421-1425

Zhong JJ (2002) Plant cell culture for production of paclitaxel and other taxanes. J Biosci Bioeng 94:591-599 